\title{
Estudo da eficácia do tratamento coadjuvante de Ameloblastoma com Solução de Carnoy
}

\author{
Study of the Effectiveness of the Co-Adjuvant \\ Treatment of Ameloblastoma With Carroy Solution
}

\author{
VITOR FIgUEIREDO DE ALMEIDA FERRER' \\ ISADORA OLIVEIRA CORRÊA" \\ EUGÊNIO BRAZ RODRIGUES ABRANTES ${ }^{\prime \prime \prime}$ \\ RODRIGO FIGUEIREDO DE BRITO RESENDE IV I
}

\begin{abstract}
RESUMO
Objetivo: Realizar uma revisão da literatura descrevendo o uso da solução de Carnoy como método coadjuvante no tratamento conservador de Ameloblastoma. Métodos: Foram selecionados artigos em português, inglês e espanhol, de janeiro de 2000 até janeiro de 2019, nos bancos de dados da Scielo, ScienceDirect, PubMed/MEDLINE e BVS. Resultados: Foram encontrados 278 artigos e selecionados sete de acordo com os critérios de inclusão e exclusão. Conclusão: O uso da solução de Carnoy como método coadjuvante no tratamento de lesões como o Ameloblastoma se mostra promissor na prevenção de recidivas, porém, sua eficácia ainda necessita de estudos mais aprofundados e prolongados.
\end{abstract}

Palavras-chave: Ameloblastoma. Solução de Carnoy. Tratamento conservador.

\begin{abstract}
Objective: To perform a literature review describing the use of Carnoy's solution as an adjuvant method in conservative treatment of Ameloblastoma. Methods: Articles were selected in Portuguese, English and Spanish, from January 2000 to January 2019, in Scielo, ScienceDirect, PubMed / MEDLINE and VHL databases. Results: We found 278 articles and selected seven according to those with inclusion and exclusion criteria. Conclusion: The use of Carnoy's solution as an adjunctive method in the treatment of lesions such as Ameloblastoma is promising in preventing relapse, but its efficacy still needs further and longer studies. Key-words: Ameloblastoma. Carnoy's solution. Cconservative treatment.
\end{abstract}

Cirurgião-dentista pela Faculdade de Odontologia da Universidade Federal Fluminense, Niterói, Rio de Janeiro/RJ, Brasi

Cirurgiã-dentista pela Faculdade de Odontologia da Universidade Federal Fluminense, Niterói, Rio de Janeiro/RJ, Brasil.

III Cirurgião-dentista, especialista em Estomatologia pela Universidade Federal do Rio de Janeiro e Residente do Serviço de Cirurgia e Traumatologia Bucomaxilofacial da Universidade Federal Fluminense/Hospital Federal dos Servidores do Estado, Rio de Janeiro/RJ, Brasil.

Iv Cirurgião-dentista, especialista em Cirurgia e Traumatologia Bucomaxilofacial pela Universidade Gama Filho/Hospital de Força Aérea do Galeão, Rio de Janeiro/RJ, Brasil. Mestre e Doutor em Odontologia pela Universidade Federal Fluminense, Niterói/RJ, Brasil. 


\section{INTRODUÇÃO}

O ameloblastoma é um tumor odontogênico benigno, que apresenta comportamento agressivo nos maxilares. ${ }^{1}$ Sua incidência ocorre na terceira e quarta décadas de vida, e a proporção entre homens e mulheres é de 1:1, sendo relativamente raro em crianças e jovens. ${ }^{2,3}$

O ameloblastoma pode ser classificado de acordo com suas características clínicas e radiográficas, em sólido ou multicístico, unicístico e periférico e, histopatologicamente, apresenta as classificações folicular, plexiforme, acantomatoso, padrão de células granulares, desmoplásico e padrão de células basais. ${ }^{4}$

O ameloblastoma sólido é o tipo mais comum e mais agressivo que se desenvolve de forma assintomática, com maior prevalência na terceira idade e com uma aparência radiográfica compatível sugestivamente conhecida como "bolhas de sabão". O ameloblastoma unicístico acomete mais a região da mandíbula, em pacientes mais jovens sem sintomatologia, radiograficamente se caracteriza por uma imagem radiolúcida circunscrita bem delimitada por um halo radiopaco, sugerindo uma lesão de origem cística. $\mathrm{O}$ ameloblastoma periférico acomete indivíduos de meia-idade e é identificado como uma lesão extraóssea, localizado na mucosa alveolar e gengiva posteriores da mandíbula, essas características clínicas não são patognomônicas, podendo gerar diagnóstico diferencial com lesões como fibroma traumático ou granuloma piogênico. ${ }^{5}$

Os tratamentos conservadores estão associados a uma morbidade mínima, mesmo com os subtipos unicísticos e periféricos menos agressivos. Em lesões agressivas essas abordagens, como crioterapia, radioterapia, enucleação seguida de curetagem, ostectomia periférica e irrigação com solução de Carnoy, entretanto, estão associadas a taxas de recorrência mais elevada, já que seu tratamento por curetagem pode deixar resquícios de pequenas ilhas de tecido tumoral residual no osso periférico, levando à necessidade de procedimentos repetidos. ${ }^{6}$ Por sua vez, a mandibulectomia segmentar radical que preconiza margem de ressecção de 15-20 mm, indicada para o tipo sólido ou multicístico, apesar de estar associada a taxas muito baixas de recidiva, apresenta como desafio a reconstrução. ${ }^{7}$

O índice de recidiva nos casos de enucleação associada ao uso da solução de Carnoy é consideravelmente menor quando comparada aos casos de enucleação isolada das lesões com componente cístico. O mecanismo de ação da Solução de Carnoy consiste em provocar uma necrose óssea periférica e, assim, promover taxas menores de recidiva., ${ }^{8,9}$ As principais complicações da solução de Carnoy são deiscência, infecção, formação de sequestros ósseos e parestesia, consequência do contato direto da substância com o tecido neural. ${ }^{8} \mathrm{O}$ tempo desse contato parece crucial para a lesão ao nervo, podendo apresentar dano após 2-3 minutos de exposição. ${ }^{10,11}$

O propósito deste artigo é realizar uma revisão da literatura analisando as características clínicas e histológicas de ameloblastomas tratados coadjuvantemente com a solução de Carnoy e discutir a eficiência desse tratamento alternativo.

\section{MATERIAIS E MÉTODOS}

Foi realizada uma revisão da literatura com o objetivo de avaliar características clínicas e o índice de recorrência dos relatos de casos de Ameloblastoma tratados com enucleação e solução de Carnoy. No levantamento bibliográfico foram feitas pesquisas nas bases de dados dos Literature Analysis (PubMed/ MEDLINE), Scientific Electronic Library Online (SciELO), BVS e ScienceDirect (Tabela 1) com os seguintes critérios de inclusão: relatos de casos publicados, entre o período do janeiro de 2000 até janeiro de 2019, nos idiomas português, 
inglês e espanhol com informações sobre o tratamento, possuindo resumo disponível. Os critérios de exclusão utilizados foram: pesquisas realizadas em animais, artigos em outra língua que não o inglês, português e espanhol, revisões de literatura sem relato de caso associado. Os termos usados para a busca foram: ameloblastoma (ameloblastoma), conservative treatment (tratamento conservador), Carnoy's solution (solução de Carnoy).

Tabela 1. O fluxograma mostra o resultado dos cruzamentos realizados nas bases de dados.

\begin{tabular}{|c|c|c|c|c|c|}
\hline Base de dados & Termos de busca & Achados & Excluído & $\begin{array}{l}\text { Exclusão por } \\
\text { repetição }\end{array}$ & Incluído \\
\hline \multirow{2}{*}{ SciELO } & $\begin{array}{l}\text { ameloblastoma } X \text { tratamento conservador } \\
\text { X solução de Carnoy }\end{array}$ & 0 & - & 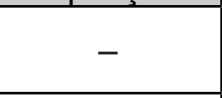 & - \\
\hline & ameloblastoma X solução de Carnoy & 1 & 1 & 0 & 0 \\
\hline \multirow{2}{*}{ PubMed } & $\begin{array}{l}\text { ameloblastoma } X \text { tratamento conservador } \\
X \text { solução de Carnoy }\end{array}$ & 5 & 2 & 0 & 3 \\
\hline & ameloblastoma X solução de Carnoy & 17 & 10 & 3 & 4 \\
\hline \multirow{2}{*}{ ScienceDirect } & $\begin{array}{l}\text { ameloblastoma } X \text { tratamento conservador } \\
X \text { solução de Carnoy }\end{array}$ & 101 & 99 & 2 & 0 \\
\hline & ameloblastoma X solução de Carnoy & 163 & 159 & 4 & 0 \\
\hline \multirow{2}{*}{ BVS } & $\begin{array}{l}\text { ameloblastoma X tratamento conservador } \\
\text { X solução de Carnoy }\end{array}$ & 5 & 2 & 3 & 0 \\
\hline & ameloblastoma X solução de Carnoy & 18 & 11 & 7 & 0 \\
\hline & & & & TOTAL: & 7 \\
\hline
\end{tabular}

\section{RESULTADOS}

Foram encontrados 278 artigos, entretanto, ancorado nos critérios de inclusão e exclusão, e após a leitura dos resumos, foram selecionados apenas sete artigos e nestes constam 67 relatos de casos de ameloblastomas tratados com enucleação e solução de Carnoy. Na Tabela 2, estão apresentados os artigos, contendo informações sobre classificação do ameloblastoma, forma de tratamento e acompanhamento. Apesar da busca dos artigos atingir artigos em português, inglês e espanhol, a totalidade dos artigos incluídos está na língua inglesa. $\mathrm{Na}$ Tabela 3, foram expostos as faixas etárias e sexo dos pacientes, assim como, a localização da lesão em questão.

Dos trabalhos selecionados, oito apresentaram recorrências da lesão após o tratamento com enucleação e solução de Carnoy, correspondendo a $11 \%$ da totalidade dos casos levantados. Dos trabalhos mostrando recidiva da lesão, cinco foram diagnosticados como lesões de padrão unicístico. Entre os trabalhos que descreveram precisamente as características físicas dos pacientes, a relação homem-mulher foi de 5:3 e a média de idade chegou a 27,5 anos. A grande maioria das lesões estava localizada na mandíbula, sendo a região posterior e o ramo os mais afetados. 
Vitor Figueiredo de Almeida Ferrer et al.

Tabela 2. Trabalhos selecionados seguindo os critérios de inclusão, indicando as características do tratamento.

\begin{tabular}{|c|c|c|c|c|c|c|c|c|}
\hline $\mathbf{N}^{\circ}$. & Autor/Ano & $\begin{array}{c}\mathrm{N}^{\circ} . \\
\text { Casos }\end{array}$ & $\begin{array}{l}\text { Casos } \\
\text { Aceitos }\end{array}$ & Classificação & Tratamento & $\begin{array}{c}\text { Exposição } \\
\text { (Carnoy) }\end{array}$ & Follow-up & Recorrência $^{\circ}$ \\
\hline 1 & $\begin{array}{c}\text { Haq et al. }{ }^{12} \\
(2016)\end{array}$ & 27 & 25 & $\begin{array}{l}\text { (4) unicístico } \\
\text { (2) plexiforme/folicular } \\
\text { (3) plexiforme } \\
\text { (1) folicular } \\
\text { (15) sólido/multicístico }\end{array}$ & $\begin{array}{c}\text { Enucleação } \\
+C\end{array}$ & $\begin{array}{l}3 \mathrm{~min} / 2 \\
\text { vezes }\end{array}$ & $\begin{array}{c}3 \text { anos (média) } \\
3-156\end{array}$ & $\begin{array}{l}\text { (1) Unicístico } \\
(2) \\
\text { Sólido/multicístico }\end{array}$ \\
\hline 2 & $\begin{array}{l}\text { Naidu et al. }{ }^{13} \\
\text { (2014) }\end{array}$ & 1 & 1 & (1) Unicístico & $\begin{array}{c}\text { Enucleação } \\
+C\end{array}$ & $5 \mathrm{~min}$ & Indefinido & $\mathrm{NI}$ \\
\hline 3 & $\begin{array}{c}\text { Kalaskar et al. }{ }^{14} \\
\text { (2011) }\end{array}$ & 2 & 2 & (2) Unicístico & $\begin{array}{c}\text { Enucleação } \\
+C\end{array}$ & $3 \min$ & Indefinido & $\mathrm{NI}$ \\
\hline 4 & $\begin{array}{l}\text { Seintou et al. }{ }^{15} \\
\qquad(2014)\end{array}$ & 51 & 1 & (1) Unicístico Folicular & $\begin{array}{c}\text { Enucleação } \\
+ \text { C }\end{array}$ & $\mathrm{NI}$ & 5 anos & $\mathrm{NI}$ \\
\hline 5 & $\begin{array}{l}\text { Rajeshkumar et } \\
\qquad \text { al. }^{16} \\
(2013)\end{array}$ & 12 & 4 & $\begin{array}{l}\text { (2) Unicístico } \\
\text { (1) Acantomatoso } \\
\text { (1) Plexiforme }\end{array}$ & $\begin{array}{c}\text { Enucleação } \\
+ \text { C }\end{array}$ & $3 \min$ & $\begin{array}{c}15 \text { meses } \\
\text { (média) } \\
6-36\end{array}$ & Nenhum \\
\hline 6 & $\begin{array}{l}\text { Chapelle et al. }{ }^{17} \\
\qquad(2004)\end{array}$ & 19 & 8 & $\begin{array}{l}\text { (1) Unicístico Grupo 2* } \\
\text { (3) Multicístico Folicular } \\
\text { (3) Unicístico Grupo 3* } \\
\text { (1) Multicístico }\end{array}$ & $\begin{array}{c}\text { Enucleação } \\
\qquad+\mathrm{C}\end{array}$ & $\mathrm{NI}$ & $\begin{array}{c}9,5 \text { meses } \\
\text { (média) } \\
2-20\end{array}$ & $\begin{array}{l}\text { (1) Multicístico } \\
\text { Folicular }\end{array}$ \\
\hline 7 & $\begin{array}{l}\text { Lee et al. }{ }^{18} \\
\qquad(2004)\end{array}$ & 29 & 22 & (22) Unicístico & $\begin{array}{c}\text { Enucleação } \\
+ \text { C }\end{array}$ & $3 \min$ & $\begin{array}{l}6.2 \text { anos } \\
\text { (média) }\end{array}$ & (4) Unicístico \\
\hline
\end{tabular}

Legenda: (C) Solução de Carnoy, (NI) não informado. 
Tabela 3. Características clínicas da lesão e dos pacientes relatados.

\begin{tabular}{|c|c|c|c|c|}
\hline $\mathrm{N}^{\circ}$. & Autor/Ano & Sexo & Idade & Localização da Lesão \\
\hline 1 & Haq et al. ${ }^{12}$ & $\begin{array}{l}\text { (13) M } \\
(12) F\end{array}$ & $\mathrm{NI}$ & $\begin{array}{l}\text { Um total de } 8 \text { (30\%) envolveu o corpo / ramo esquerdo, } 10 \\
(37 \%) \text { o corpo / ramo direitos, e } 9 \text { (33\%) envolveram a região } \\
\text { parassinfisária ou cruzaram a linha média. }\end{array}$ \\
\hline 2 & Naidu et al. ${ }^{13}$ & $\mathrm{~F}$ & 11 & Corpo de ramo de mandíbula \\
\hline \multirow{2}{*}{3} & \multirow{2}{*}{ Kalaskar et al. ${ }^{14}$} & $\mathrm{~F}$ & 12 & Região de molares inferiores- ramo mandibular \\
\hline & & M & 9 & Região de canino, $1^{\circ}$. e $2^{\circ}$. molares superiores \\
\hline 4 & Seintou et al. ${ }^{15}$ & M & 13 & Ramo e processo coronoide \\
\hline \multirow{4}{*}{5} & \multirow{4}{*}{ Rajeshkumar et al. ${ }^{16}$} & M & 60 & Área de mandíbula posterior (extensão até o ramo) \\
\hline & & M & 65 & Área de mandíbula posterior \\
\hline & & M & 9 & Área de ângulo de mandíbula \\
\hline & & M & 30 & Área de mandíbula anterior \\
\hline \multirow{8}{*}{6} & \multirow{8}{*}{ Chapelle et al. ${ }^{17}$} & M & 18 & \multirow{8}{*}{$\begin{array}{l}\text { Proporção de } 18: 1 \text { entre mandíbula e maxila entre os } 19 \text { relatos } \\
\text { de casos presente no artigo. Sendo a região posterior e o ramo } \\
\text { afetado em } 50 \%\end{array}$} \\
\hline & & $\mathrm{F}$ & 17 & \\
\hline & & M & 17 & \\
\hline & & $\mathrm{F}$ & 27 & \\
\hline & & $\mathrm{F}$ & 33 & \\
\hline & & M & 31 & \\
\hline & & M & 53 & \\
\hline & & $F$ & 35 & \\
\hline 7 & Lee et al. ${ }^{18}$ & $\begin{array}{c}12 \text { homens e } 17 \\
\text { mulheres }\end{array}$ & $\begin{array}{l}\text { Faixa etária de } \\
\text { 13-50 anos (idade } \\
\text { mediana de } 23\end{array}$ & $\begin{array}{l}\text { A maioria das lesões ( } 86 \% \text { ) ocorreu em mandíbula e das quatro } \\
\text { lesões em a maxila, dois apresentados na maxila posterior. Mais } \\
\text { da metade das lesões mandibulares também estavam na região } \\
\text { perto do ângulo e ascendente ramus, } 24 \% \text { ocorreram no corpo } \\
\text { de mandíbula, e } 7 \% \text { ocorreram na região anterior da mandíbula. }\end{array}$ \\
\hline
\end{tabular}

\section{DIscussão}

Entre as neoplasias odontogênicas, o ameloblastoma corresponde a uma patologia que necessita de grande atenção devido a sua prevalência, comportamento agressivo e o tra- tamento em constante discussão entre os cirurgiões. $^{5}$

Em nosso levantamento bibliográfico, ocorreu uma proporção maior de relatos de caso em criança, o que difere da incidência etária dessa patologia. ${ }^{19}$ Esse cenário pode 
ser explicado pela tendência conservadora no tratamento de ameloblastoma em crianças, uma vez que nessa idade o tratamento é dificultado devido ao contínuo crescimento facial, a diferença da fisiologia óssea, presença de dentes não erupcionados, dificuldade inicial do diagnóstico e predominância do ameloblastoma unicístico, tornando o tratamento mais difícil. ${ }^{13,20}$

A classificação do ameloblastoma é importante, pois o tratamento de escolha é fundamentado no diagnóstico histopatológico. Entretanto, Haq et al. ${ }^{12}$ relataram em sua série de casos a alta taxa de mudança entre o diagnóstico inicial e as variantes de ameloblastoma após avaliação radiográfica, exame clínico e principalmente após o tratamento definitivo e remoção da peça cirúrgica completa. Com isso, o manejo cirúrgico, ancorado no diagnóstico inicial, pode não ser o mais eficiente para algumas lesões.

Entre os 67 casos relatados nesses artigos, 55\% foram classificados como Ameloblastoma Unicístico (AU). Ackermann et al. ${ }^{21}$ subdividiram o AU seguindo o aspecto histológico em luminal, intraluminal e mural. Chapelle et al. ${ }^{17}$ propuseram nos casos de AU intraluminal e luminal intervenção radical somente se houver recorrência, entretanto nos casos de AU mural realizar intervenção cirúrgica mais radical, como ressecção cirúrgica completa. $\mathrm{O}$ mesmo autor relatou a eficiência do tratamento de AU com Carnoy, e atribuiu parte das recorrências à mudança entre o diagnóstico inicial e o histopatológico. Já Pogrel, em 2009, recomendou que o tratamento de Ameloblastoma ancorado em enucleação associado à Solução de Carnoy ou nitrogênio líquido pode apresentar bons resultados, uma vez que o tratamento limitado a enucleação mostra uma taxa de $60 \%$ de recorrência. ${ }^{22}$

Proporcionalmente, a maior taxa de recidiva em nosso levantamento foi do Ameloblastoma em sua classificação multicística.
Isso justifica a grande discussão em torno do tratamento dessa condição, que para muitos cirurgiões deve ser abordada com tratamento radical devido a sua agressividade. ${ }^{23}$ Chapelle et al. ${ }^{17}$ e Haq et al. ${ }^{12}$ relataram abordagem conservadora em Ameloblastoma Sólido, porém ambos ressaltaram a agressividade dessa lesão. Chapelle et al. ${ }^{17}$ sugeriram um tratamento radical nesses casos. Já Haq et al. ${ }^{12}$ confirmaram a tendência de tratamento radical, porém em crianças salientaram a possibilidade de realizar uma abordagem conservadora. Além disso, Haq et al. ${ }^{12}$ ressaltaram que na escolha de uma abordagem conservadora deve-se levar em consideração a localização da lesão, em região de maxila parece arriscado devido à evolução para áreas de difícil acesso e próxima a estruturas nobres, como a base do crânio.

A solução Carnoy é composta por álcool, ácido acético glacial, clorofórmio e cloreto férrico e a aplicação da mesma diminui a recorrência da lesão após a enucleação. ${ }^{23,24,25}$ No entanto, o clorofórmio foi retirado da composição por ser considerado carcinogênico, ${ }^{23,24,}$ o que deu origem à solução Carnoy modificada, que apresenta maiores taxas de recorrência. ${ }^{23}$ Entre os sete artigos selecionados, apenas três descrevem a composição da solução Carnoy, ${ }^{12,16,18}$ sendo que há uma contraposição com outras pesquisas em relação ao componente cancerígeno. Em março de 2013, Rajeshkumar et al. ${ }^{16}$ afirmaram que o cloreto férrico é responsável pelo teor de malignidade da composição da solução Carnoy e por isso não está mais na fórmula. Em 1931, surgiram os primeiros relatos do uso de solução Carnoy em tratamento de cistos odontogênicos. ${ }^{26}$ Os primeiros registros para o uso de solução Carnoy para complementação de tratamento de ameloblastoma unicístico surgiram em 1987 por Stoelinga e Brokenhort et al. ${ }^{18}$. Em janeiro de 2012, Kalaskar et al. ${ }^{14}$ afirmaram que o diagnóstico preciso com intervenção e tratamento cirúrgico radical correto, seguido de aplicação 
de solução Carnoy podem melhorar o prognóstico e complicações associadas a ressecções.

Alguns cuidados devem ser mantidos quando se trata do uso da solução de Carnoy. ${ }^{12,13,14,18}$ Frerich et al., ${ }^{10}$ em seu estudo pré-clínico, sugeriram que a aplicação de Carnoy não deve ultrapassar três minutos sem contato direto com substâncias e tecidos adjacentes, isso diminui a chance de maiores complicações. A literatura preconiza que se deve ter atenção para a solução não ser aplicada diretamente sobre tecidos nervosos, pois isso acarretaria danos como parestesia, sensação de queimação ou perda total da sensibilidade. ${ }^{14}$ Entre os artigos selecionados, apenas dois artigos ${ }^{14,16}$ não citam o tempo de aplicação da solução e os cuidados necessários para o seu uso. Saulacic et al. ${ }^{27}$ levantaram a discussão acerca do efeito da exposição de vasos sanguíneos à solução, e, mesmo ocorrendo danos, como a perda focal do endotélio, o mesmo se recuperaria quando a aplicação não ultrapassa cinco minutos. Outras complicações e efeitos colaterais foram apresentados por diversos autores, como infecção e formação de sequestros ósseos, porém, a literatura é pouco descritiva a respeito dessas condições. ${ }^{10}$ Mesmo diante de seus efeitos adversos, a solução de Carnoy, se torna opção de escolha para tratamentos em áreas nobres e de difícil acesso, esses tratamentos invasivos são descartados, ou em regiões próximas a estruturas dentárias, de lesões com grande extensão.

\section{CONSIDERAÇõES FINAIS}

Tratamentos conservadores de lesões odontogênicas são com frequência estudados por pesquisadores e cirurgiões com o intuito de eliminar as dificuldades da reabilitação subsequente imposta por tratamentos radicais. A solução de Carnoy, quando aplicada corretamente em lesões de características císticas e de poder de infiltração diminuído, não apre- senta altos índices de complicações e mostra uma tendência à diminuição de recorrências. Entretanto, em lesões de curso invasivo deve-se levar em consideração a maior tendência de recorrência quando a escolha é o tratamento conservador.

\section{REFERÊNCIAS}

1. Philipsen HP, Reichart PA. Classification of odontogenic tumours. A historical review. Journal of oral pathology \& medicine: official publication of the J Oral Pathol Med. 2006; 35: 525-529.https://www.ncbi.nlm.nih.gov/pub$\mathrm{med} / 16968232$

2. Gunhan O., Erseven G., Ruacan S., Celasun B., Aydintug Y., Ergun E., et al. Odontogenic tumours. A series of 409 cases. Aust Dent J. 1990; 35: 518-522. https://www.ncbi.nlm.nih. gov/pubmed/2090083

3. Hirschhorn Al, Vered M., Buchner A., Greenberg G., Yahalom R. Unicystic ameloblastoma in an infant: a management dilemma. J Craniomaxillofac Surg. 2013; 41: 226-230.https:// www.ncbi.nlm.nih.gov/pubmed/23402730

4. Bachmann AM, Linfesty RL. Ameloblastoma, solid/multicystic type. Head Neck Pathol. 2009; 3: 307-309.https://www.ncbi.nlm.nih.gov/pmc/ articles/PMC2811572/

5. Neville BW, Damm DD, Allen CM, Bouquot JE. Patologia Oral e Maxilofacial. Trad. 3. ed., Rio de Janeiro: Elsevier; 2009, p. 703-711.

6. Montoro JR, Tavares MG, Melo DH, Franco R. de L., Mello-Filho FV, Xavier SP, et al. Mandibular ameloblastoma treated by bone resection and immediate reconstruction. Braz J Otorhinolaryngol. 2008; 74: 155-157.https://www. ncbi.nlm.nih.gov/pubmed/18392519

7. Ooi A., Feng J., Tan HK, Ong YS. Primary treatment of mandibular ameloblastoma with segmental resection and free fibula reconstruction: achieving satisfactory outcomes with low implant-prosthetic rehabilitation uptake. Journal of Plastic, Reconstructive \& Aesthetic Surgery. 2014; 67 (4): 498-505.https://www.ncbi. nlm.nih.gov/pubmed/24508227 
Vitor Figueiredo de Almeida Ferrer et al.

8. Ribeiro Júnior O., Borba AM, Brozoski MA, Martins R., Alves ACF, Naclério-Homem MG. Importância dos tratamentos complementares na terapêutica cirúrgica do tumor odontogênico queratocístico. RPG Rev Pós Grad. 2012; 19 (3): 122-8.http://revodonto.bvsalud. org/scielo.php?script=sci_arttext\&pid=S0104-56952012000300006\&lng=pt\&nrm=iso

9. Ecker J., Horst R., \& Koslovsky D. Current Role of Carnoy's Solution in Treating Keratocystic Odontogenic Tumors. Journal of Oral and Maxillofacial Surgery. 2016; 74 (2): 278-282.https:// www.ncbi.nlm.nih.gov/pubmed/26272006

10. Frerich B., Cornelius CP, \& Wiethölter H. Critical time of exposure of the rabbit inferior alveolar nerve to Carnoy's solution. Journal of Oral and Maxillofacial Surgery. 1994; 52 (6): 599-606. https://www.ncbi.nlm.nih.gov/pubmed/8189298

11. Freedman M., Stassen LF. Commonly used topical oral wound dressing materials in dental and surgical practice - a literature review. J Ir Dent Assoc. 2013, Aug./Sep.; 59 (4): 190-195.https:// www.ncbi.nlm.nih.gov/pubmed/24156211

12. Haq J., Siddiqui S., McGurk M. Argument for the conservative management of mandibular ameloblastomas. Br J Oral Maxillofac Surg. 2016; 54: 1.001-1.005.https://www.ncbi.nlm.nih.gov/ pubmed/27599408

13. Naidu SR, Hegde RJ, Devrukhkar VN, Patel AR. Conservative management of unicystic ameloblastoma in a young child: a case report. Journal of Indian Society of Pedodontics and Preventive Dentistry. 2014; 32 (3): 251-254. http://www.jisppd.com/article.asp?issn=09704388; year $=2014 ;$ volume $=32 ;$ issue $=3 ;$ spage $=25$ 1 ; epage $=254$; aulast $=$ Naidu

14. Kalaskar R., Unawane AS, Kalaskar AR, Pandilwar $P$. Conservative management of unicystic ameloblastoma in a young child: Report of two cases. Contemp Clin Dent 2011; 2: 359-63. https://www.ncbi.nlm.nih.gov/ pubmed/22346168

15. Seintou A., Martinelli-Kläy CP, Lombardi T. Unicystic ameloblastoma in children: systematic review of clinicopathological features and treatment outcomes. Int $\mathbf{J}$ Oral Maxillofac Surg. 2014, Apr.; 43 (4): 405-12.https://www.ncbi.nIm. nih.gov/pubmed/24503101
16. Rajeshkumar BP, Rai KK, Geetha NT, Shivakumar HR, Upasi AP. Carnoy's in Aggressive Lesions: Our Experience. J Maxillofac Oral Surg. 2013; 12: 42-47.https://www.ncbi.nlm.nih.gov/ pubmed/24431812

17. Chapelle KA, Stoelinga PJ, de Wilde PC, Brouns JJ, Voorsmit RA. Rational approach to diagnosis and treatment of ameloblastomas and odontogenic keratocysts. Br J Oral Maxillofac Surg. 2004; 42 (5): 381-90.https://www.ncbi.nlm.nih. gov/pubmed/15336762

18. Lee PK, Samman N., Ng IO. Unicystic ameloblastoma - use of Carnoy's solution after enucleation. Int J Oral Maxillofac Surg. 2004; 33: 263-7.https://www.ncbi.nlm.nih.gov/ pubmed/15290793

19. Baldasserini G., Scomparin L., Freitas KMS, Souza DFM, Cardoso R., Paredes WEB. Epidemiological profile of ameloblastoma affected patients subjected to surgery at a tertiary hospital in the state of São Paulo. Rev. Odont. Mex [on-line], 2018; 22 (2): 82-87.http://www.scielo. org.mx/pdf/rom/v22n2/1870-199X-rom-22-0282-en.pdf

20. Guerrisi M., Piloni MJ, Keszler A. Odontogenic tumors in children and adolescents. A 15-year retrospective study in Argentina. Med Oral Patol Oral Cir Bucal. 2007; 12: 180-185.http:// www.medicinaoral.com/pubmed/medoralv12 i3_p180.pdf

21. Ackermann GI, Altini M., Shear M. The unicystic ameloblastoma: a clinicopathological study of 57 cases. J Oral Pathol. 1988; 17 (9-10): 541-6. https://www.ncbi.nlm.nih.gov/pubmed/3150441

22. Pogrel MA, \& Montes DM. (2009). Is there a role for enucleation in the management of ameloblastoma? International Journal of Oral and Maxillofacial Surgery. 2009; 38 (8): $\quad$ 807-812.https://www.ncbi.nlm.nih.gov/ pubmed/19297131

23. Hendra FN, Cann V, Helder EM, Ruslin MN, de Visscher JG, Forouzanfar T., Vet HCW. Global incidence and profile of ameloblastoma: a systematic review and meta-analysis. Oral Diseases. 2019; 00: 1-10.https://onlinelibrary.wiley. com/doi/abs/10.1111/odi.13031

24. Ledderhof NJ, Caminiti MF, Bradley GL. Topical 5-Fluorouracil is a Novel Targeted Therapy 
for the Keratocystic Odontogenic Tumor. Journal of Oral and Maxillofacial Surgery. 2017; 75 (3): 514-524.https://www.joms.org/article/ S0278-2391(16)30899-0/fulltext

25. Carvalho FSR, Feitosa VP, Silva PGB, Soares ECS, Ribeiro TR, Fonteles CSR, \& Costa FWG. Evaluation of different therapeutic Carnoy's formulations on hard human tissues: A Raman microspectroscopy, microhardness, and scanning electron microscopy study. Journal of Cranio-Maxillofacial Surgery. 2018; 46 (5): 749-758.http://www.repositorio.ufc.br/bitstream/ riufc/31658/1/2018_art_fsrcarvalho.pdf
26. Hellstein J., Hopkins T., Morgan T. The history and mystery of Carnoy's solution: an assessment of the need for chloroform. Oral Surg Oral Med Oral Pathol Oral Radiol Endod 2007; 103: 24.https://www.oooojournal.net/article/ S1079-2104(07)00050-9/abstract

27. Saulacic N., Stajcic Z., Stajcic LS, Piattelli A., lizuka T., \& Lombardi T. Effects of Carnoy's solution on blood vessels of the axillary fossa of rats. International Journal of Oral and Maxillofacial Surgery. 2009; 38 (8): 876-879.https:// www.ncbi.nlm.nih.gov/pubmed/19427171

Submetido em: 29-7-2019

Aceito em: 5-3-2020 